\title{
The construction problem in Kähler geometry
}

Carlos Simpson,

CNRS, Laboratoire J.A. Dieudonné

Université de Nice-Sophia Antipolis

Parc Valrose, 06100 Nice

carlos@math.unice.fr

Dedicated to Alexander Reznikov

One of the most surprising things in algebraic geometry is the fact that algebraic varieties over the complex numbers benefit from a collection of metric properties which strongly influence their topological and geometric shapes. The existence of a Kähler metric leads to all sorts of Hodge theoretical restrictions on the homotopy types of algebraic varieties. On the other hand, a sparse collection of examples shows that the remaining liberty is nontrivially large. Paradoxically, with all of this information, the research field remains as wide open as it was many decades ago, because the gap between the known restrictions, and the known examples of what can occur, only seems to grow wider and wider the more closely we look at it.

In spite of the differential-geometric nature of the questions and methods, the origins of the situation are very algebraic. We look at subvarieties of projective space over the complex numbers. The main over-arching problem in algebraic geometry is to understand the classification of algebro-geometric objects. The topology of the usual complex-valued points of a variety plays an important role, because the topological type is a locally constant function on any classifying space. Thus the partitioning of the classification problem according to topological type provides a coarse, and more calculable, alternative to the partition by connected components. Furthermore, the topology of a variety strongly influences its geometric properties. A sociological observation is that the quest for understanding the topology of algebraic varieties has led to a rich set of techniques which found applications elsewhere, even in physics.

Perhaps a word about the choice of ground field is appropriate. One could also look at the shapes of the real points of real algebraic varieties. However, by Weierstrass approximation, pretty much anything can arise if you let the degree get big enough. Thus the classical question in this case is "which shapes can occur for a given degree?" This is so much more difficult 
that it constitutes a separate subject. ${ }^{1}$ Taking the algebraically closed field of complexes means that we don't unwittingly leave any points out of the picture, so it seems reasonable to regard this as one possible canonical choice. Historically at least, and probably also for some philosophical reasons, investigations into the topology of complex varieties have in turn been mirrored in arithmetic algebraic geometry over other fields. A lot of what we say for complex Hodge theory could also apply to the $p$-adic versions.

It is a standard norm for mathematical papers, to discuss positive contributions to the collective knowledge. I would like to take advantage of the present opportunity, to focus on a zone where we have little or no knowledge, and where I have nothing new to report, in any case. I apologize therefore for the very vague nature of most of the discussion. I hope that the references $^{2}$ will give readers a place to look for whatever details are available. With our purpose stated this way, we should normally try to cover most of modern Kähler geometry. However, this would be unreasonable, because for many parts of the subject I wouldn't have anything further to say than just repeating what would be in the references. So, toning down our ambitions a little bit, we get to the description of what I really would like to talk about, which is to isolate a certain family of questions which seem important, and about which very little seems to be known: how to construct algebraic (or Kähler) varieties with interesting topological behavior.

To get going, recall that projective space is endowed with the FubiniStudy metric, a hermitian metric which has the Kähler property which we now explain in two ways. The first way is to note that if you write the matrix for the hermitian metric in terms of a local system of holomorphic coordinates, then transform the tensor product between $d z_{i}$ and $d \bar{z}_{j}$ to a wedge product, you get an alternating form $\omega$. It is well-defined independantly of the choice of coordinates. The Kähler condition is that the two-form $\omega$ is closed, i.e. it gives a symplectic structure. The second definition is to say

\footnotetext{
${ }^{1}$ We can hope, however, that the intuition developped by workers in that field (do a recursive search on the references in [28]) could be of help in the complex case we are discussing here.

${ }^{2}$ In compiling the references, the online version of AMS Math Reviews was a great help in remembering, finding, organizing and writing them up (often by cut-and-paste). Readers having access to this service can greatly expand the amount of referential information available, by searching starting with the authors cited in our bibliography. A crucial recent improvement has been the inclusion of forward searching to get future papers refering to a given paper. This results in such a vast amount of information that even our rather long reference list can only be considered as a sampling.
} 
that a metric is Kähler if at each point there exists a holomorphic system of coordinates in which the matrix for the metric is equal to the identity, up to an error term in $|z|^{2}$ (rather than just $|z|$ as would be the case for an arbitrary choice of coordinates). The good system is called an osculating system of coordinates. This second point of view leads directly to the famous Kähler identities

$$
\left(D^{\prime}\right)^{*}=\sqrt{-1}\left[\Lambda, D^{\prime \prime}\right], \quad\left(D^{\prime \prime}\right)^{*}=-\sqrt{-1}\left[\Lambda, D^{\prime}\right]
$$

where $D^{\prime}=\partial$ and $D^{\prime \prime}=\bar{\partial}$ are the $(1,0)$ and $(0,1)$-components of the de Rham differential, and $\Lambda$ is the pointwise adjoint of the operation $\wedge \omega$.

The correspondence between hermitian metrics and (1,1)-forms is compatible with restriction to subvarieties, as is the Kähler condition $d \omega=0$, so any smooth subvariety $X \subset \mathbf{P}^{n}$ inherits a Kähler metric too. This extrinsic description can be modified so as to seem more intrinsic, by saying that the Kähler metric is obtained as the Chern $(1,1)$-form of a positively curved metric on a line bundle $L$ over $X$ : in the projective case $L$ is the pullback of the tautological bundle $\mathcal{O}_{\mathbf{P}^{n}}(1)$ on projective space. In a philosophical sense, a Kähler metric should be thought of as corresponding to a curvature of a connection on a line bundle; this will only be precise if the cohomology class $[\omega]$ is integral. On the other hand, there may be algebraic varieties, or other more exotic things such as algebraic spaces, which don't have projective embeddings and so aren't Kähler. Nonetheless, the main intention of the theory is to investigate the properties of projective varieties. There are some significant differences between the Kähler and the projective situations [194], but in spite of those we will often mix things up, so that questions posed for one class of varieties might well be reinterpreted for any other related class.

When a variety $X$ has a Kähler metric $\omega$, the Kähler identities imply that the Laplacians for $d$ and $D^{\prime \prime}$ are proportional. This leads ${ }^{3}$ to the Hodge decomposition for cohomology (certainly when $X$ is compact, and some results are possible in the noncompact case too [63] [156] [183] [201]). It gives us a first Hodge-theoretic result about the homotopy of $X$ : if $i$ is odd then $b_{i}(X):=\operatorname{dim} H^{i}(X, \mathbf{C})$ is even. ${ }^{4}$ Johnson and Rees point out that this result carries over easily to the case of cohomology with coeficients in a local system

\footnotetext{
[27].

${ }^{3}$ Some good references for the theory starting from the beginning are [87], [194], [195],

${ }^{4}$ This apparently characterizes exactly those algebraic surfaces which are projective, as was stated in Simanca's Math Review MR 1723831 of [10] but I didn't find the actual reference for it.
} 
with finite monodromy group [104], and they use it to show that certain free products cannot occur. This was extended by Arapura [11] to cover certain amalgamated products, in a more elementary version of Gromov' general theorem that $\pi_{1}(X)$ can never be a nontrivial amalgamated product [88].

Treating the noncompact case by compactifying, resolving singularities, ${ }^{5}$ and then using the logarithmic de Rham complex, leads to mixed Hodge structures on the cohomology in this case [63] [183] [201] [173] [172] [149]. Combining with simplicial techniques gives mixed Hodge structures on singular varieties too.

A classical technique for analyzing the topology of algebraic varieties is Lefschetz devissage [63]. One can arrange (by a birational transformation) to have a fibration $X \rightarrow \mathbf{P}^{1}$ whose fibers are $n$-1-dimensional varieties, a finite number of them singular (that is to say more singular than the others). The topological properties of $X$ are determined by the topological properties of the general fiber, the way its topology is transformed under the monodromy representation of $\pi_{1}\left(\mathbf{P}^{1}-\left\{s_{1}, \ldots, s_{k}\right\}\right)$, and the way the singular fibers fill things in. This led to things such as Zariski's calculations of fundamental groups, as well as proofs of certain parts of the Lefschetz theorems (fuller and easier proofs being obtained using the Hodge decomposition for cohomology). Griffiths proposed to throw Hodge theory into this situation, namely to look at how the Hodge decomposition of the cohomology of the fibers varies. He discovered the fundamental Griffiths transversality relation governing the variance of the Hodge filtration, and developed much of the basic theory of variations of Hodge structure which shows how this family of Hodge structures integrates to give the Hodge structure on the total space. This left open the question of what happens near the singularities, the answers furnished by [174] [51]. Similarly, what happens when the total space, and hence the fibers, are noncompact and/or singular - in this case we obtain variations of mixed Hodge structure, and the combined situation where this degenerates leads to a mountain of linear algebra [183] [201] which very few can grasp (anyone out there?).

To wrap up this historical perspective a little faster, basically the remaining theoretical directions have been to look at Hodge theory for the full homotopy type of $X$. This can mean looking at the homotopy groups, which obtain mixed Hodge structures ${ }^{6}$ [141], idem for the pro-nilpotent completion

\footnotetext{
${ }^{5}$ Hironaka's result [98] has recently been revisited by several people [1] [24] [36].

${ }^{6}$ One notable exception to the principle that homotopic invariants which are finite di-
} 
of the fundamental group [141] [93]; looking at representations of $\pi_{1}(X)$ in nonabelian groups such as $G L(n)$, which have harmonic representatives [53]; and combining various of these. Much of what we shall say below issues from these basic themes.

In a family of varieties, all of the Hodge-theoretic invariants give rise to variations of mixed Hodge structure over the base. This imposes restrictions on the monodromy. We have things like the invariant cycle theorem stating that a global section which is of type $(p, q)$ at one point is also of type $(p, q)$ at other points.

A crucial property is the formality theorem of [65], saying that the cohomology ring of a simply connected smooth projective variety determines its rational homotopy type. This has been extended to the pro-nilpotent completion of $\pi_{1}(X)$ [141] [93], to the spaces of representations [144] [81], and to relative Malcev completions at other representations in [93]. In the case of open varieties, the quadratic formality condition is replaced by a weaker but still restrictive condition of generation in low degrees [141].

Any representation $\pi_{1}(X) \rightarrow G L(n, \mathbf{C})$ with reductive Zariski closure, admits a structure of harmonic bundle [53]. These behave formally much like variations of Hodge structure except that they lack a notion of "Hodge type". The space of representations of $\pi_{1}(X)$ has a hyperkähler structure,[99] [78] where the other complex structures come from the interpretation as a moduli space of Higgs bundles [99] [151] [177] [78]. The cohomologies of $X$ with coefficients in the different representations give a nicely varying family of complexes indexed by the representation space. The support loci are hyperkähler subvarieties, and even have certain rationality properties. An important distinction occurs between representations which are rigid (i.e. isolated points of the space of representations) and those which are not. Rigid representations of the fundamental group are variations of Hodge structure, and one could conjecture that they must be motivic i.e. that they should come from families of varieties (this is a special case of a slightly more general conjecture [177] (i)). On the other hand, nonrigid representations fit into a bigger factorization theory [178] [47] [202] [111] [105] [75] [117] [147] [59] which also encompasses results such as the fact (due to $L^{2}$ cohomology on infinite covers of $X$ ) that if $\pi_{1}$ decomposes into a nontrivial amalgamated sum then

mensional complex vector spaces, have mixed Hodge structures, is the case of the group cohomology of $\pi_{1}$. This was a question that was raised by many workers such as Toledo, Carlson, Hain, long ago. I don't recall whether I have heard of any progress (or counterexample) in the meantime, and Google didn't help either. 
there must be a map from $X$ to a Riemann surface of genus $\geq 2$ [88]. We now know the Shafarevich holomorphic convexity conjecture relative to reductive representations in a linear group [111] [75] [124]. There are a number of directions of investigation running which look at cohomological invariants associated to representations, specially work of Reznikov [170] which has been applied in [117]. We are starting to get a good picture of the theory of representations of the fundamental group for quasiprojective open varieties [53] [105] [138].

The above results lead to important classes of restrictions of the form, certain homotopy types or homotopical invariants, cannot occur for (projective) algebraic and/or Kähler varieties. In fact, it becomes possible to write down explicit large families of topological types which cannot occur, see [47] [178] [45] [110] [177] ... . Reznikov has proven some of the deepest versions of these restrictions, including a theorem covering many cases of a conjecture due to him, and independantly Goldman and Donaldson, saying that irreducible 3-manifold groups are not Kähler groups [170].

Intrinsic global methods appeared in the work of Calabi and Yau giving certain types of special Kähler metrics. This hooked up with a sector of the subject of interest to the physicists generating the subject of "Mirror symmetry" which can no longer be considered as just a subfield of Hodge theory or Kähler geometry. These important subjects are underrepresented in present article.

Investigations of the Hodge-theoretic properties of algebraic cycles have led very far in the direction of $K$-theory. In particular, this leads to the introduction of new invariants such as regulators [23] [167] [170], behaving in a way which is related to Hodge theory via the Beilinson conjectures and Deligne cohomology.

Recent work of Voisin has underlined the difference between the Kähler and the algebraic categories. For example, the Q-polarization of the Hodge structure of an algebraic variety is a distinguishing characteristic, the existence of which can be ruled out for certain Kähler homotopy types; this leads to Kähler homotopy types whose cohomology ring cannot be the cohomology ring of an algebraic variety $([194](\mathrm{v}))$. Philosophically going in the same direction is her result that the Hodge conjecture for representing cycles by Chern classes of bundles, cannot be true for arbitrary Kähler manifolds. Of course we don't know whether it is true for algebraic varieties, but Voisin's proof [194] (iv) clearly distinguishes these cases since it makes use of a Kähler variety (a complex torus) which has no rational Hodge classes in 
degrees 2 or 4 (in particular no hyperplane class). In view of these results, the questions of realization of homotopy types in the Kähler and projective categories are clearly distinct and can be treated as separate problems. The reader is invited to double-up our questions in this way.

It seems safe to say (if nothing else, in view of the length of the reference list which is only partial) that a relatively complete picture of the Hodgetheoretic propreties of algebraic varieties, is in the making. It would be good to understand how Lefschetz devissage works in the context of Hodge structures on homotopy, or nonabelian cohomology, see Navarro Aznar [148] for example. The theory should be extended to apply to algebraic stacks and other exotic objects. One detail for the curious, is the variance of the formality equivalence on the homotopy type given by [65]. And more globally, it seems unclear whether the full complicated version of the theory as sketched above has really been explicitly applied in many examples. Aside from these brief remarks, it is best to consult the references for a good picture of where the current development of the theory stands, and in which directions it will (or should) be developped in the near future.

Now we come to the main point of the present exposition, which is to try to isolate some of the important questions in the subject. It should be stressed that these are not at all new, but rather come from the folklore explicit or implicit in most of the work that has been done. Still, it seems like a good idea to look at them from an analytical viewpoint. A historical overview brings out an inescapable fact: we know a lot more about what (projective) algebraic, or Kähler, varieties don't look like, than about what they do. This is because the most subtle tool we have found yet is Hodge theory, and this is essentially geared toward additional properties, or equivalently, restrictions on the topology and/or geometry of Kähler manifolds. This type of application determines the style of numerous theorems in the subject. In some hypothetical alternative world, this might have been enough to gain a complete understanding of the subject, if we could prove so many properties that there were very few possibilities left and we would get a complete classification of what could happen. Unfortunately, or perhaps fortunately for the interest of the subject at least, this is not the case. In spite of the large and varied collection of properties given by Hodge theory, it seems nowhere close to giving any kind of classification of the topological behavior which could arise. 
On the other side of the story, this situation leaves wide open the whole problem of how to construct varieties exhibiting topological behavior that is allowed by Hodge theory. Theoretical machinery is not in the fore here, although it is present insofar as it facilitates the calculation of the topological behavior of a variety which for other reasons we have at hand. Unfortunately (in all senses) on this side of things, we don't have very many deep techniques of construction. ${ }^{7}$ I will try to discuss below the possible techniques of construction we know about. For the purposes of the present paragraph, let's just look at what might be seen as a good candidate for a "deep technique of construction": the notion of quotient of a symmetric domain by a group action. The associated variety comes out of a transcendental construction, so it is at least very indirect as a construction. However, the geographical place occupied by the varieties obtained in this way is quite infinitesimal, so in spite of a number of successful applications of this construction, we are mostly left wondering how come there isn't a way of twiddling it to cover more nearby cases. $^{8}$ To give a precise version of these statements, look at the case of surfaces: those which are uniformized by the ball have $c_{1}^{2}=3 c_{2}$, whereas there exist other surfaces uniformized by a product of discs, with $c_{1}^{2}=2 c_{2}$. Siu mentionned as a folkloric problem, the question of whether or not every variety with $c_{1}^{2}$ lying between $2 c_{2}$ and $3 c_{2}$, would have to have infinite fundamental group. I don't know whether it is reasonable to expect such a thing or not, but it does seem quite clear that it is silly to have such a wide swath where we think non-simply connected varieties should be lying, but to have a nice general construction only on the boundary of the region. This example is pretty emblematic of the whole problem of constructing interesting varieties: we have the impression that there is a huge mass of stuff out there, waiting to be constructed or seen, but we have no idea how to get there.

The construction question can take many forms, depending on what type of thing you want to construct. Among the infinite variations on this question, we can try to isolate some exemplary ones. Start with the simply connected case. In all of the questions, we will for simplicity ask for smooth projective varieties but the same question could be asked for proper smooth algebraic spaces, compact Kähler manifolds, etc.; and we stick to the com-

\footnotetext{
${ }^{7}$ The only full exception is the Lefschetz $(1,1)$-theorem.

${ }^{8}$ Actually, this has been done in some cases such as Toledo's examples [191], see also $[3]$
} 
pact smooth case again for simplicity, but more subtle questions involving mixed Hodge structures are available for the open and/or singular case.

The easiest version of the question would be the following: given $n$ and a vector $\left(b_{0}, \ldots, b_{2 n}\right)$ satisfying the known constraints such as $b_{k}=b_{2 n-k}$ and that $b_{2 k+1}$ is even, plus the constraint given by the Lefschetz decomposition, does there exist a smooth projective variety $X$ with $\operatorname{dim} H^{i}(X, \mathbf{C})=b_{i}$ ? A recent investigation of the very first case $b_{i}=0$ is [200]. Take note of the obvious (and in this case pretty useless) observation that there exists an algorithm which lists all of the vectors which occur: just run through all possible varieties defined over finite extensions of $\mathbf{Q}$ and for each one, calculate the Betti numbers. This does not imply that there is a decision algorithm for a given vector. To get even an impractical decision algorithm we would have to get a bound on the degree and height of varieties with a given set of Betti numbers. Of course the algorithmic question doesn't really represent what we would like, which is a clean statement saying, a vector of Betti numbers occurs if and only if it satisfies a given simple constraint.

We can refine the above question by asking for the full collection of Hodge numbers $h^{p, q}$. See [74] and [64] for example. In this case, it is interesting even to look at only one cohomology group: given a collection $\left(h^{k, 0}, \ldots, h^{0, k}\right)$ does there exist a variety $X$ such that the Hodge structure on $H^{k}(X, \mathbf{C})$ has these Hodge numbers? In a sort of vague philosophical sense, Mirror symmetry says that there may be some relationship between this Hodge number question and the Betti number question in the previous paragraph.

At the very least, there is a question which intuitively suggests itself in both cases. This was the subject of a conversation with J. Kollar a long time ago. Is it possible to have big numbers on the ends of the vector, and small numbers in the middle? Or, on the other hand, is there some type of bound that says the middle Hodge (or Betti) numbers have to be bigger than the outer ones? Our intuition says that the middle numbers probably should be bigger than the outer ones, but there doesn't seem to be any result in this direction. This can be made into a very small and specific question, for example do there exist surfaces with Hodge numbers $h^{2,0}=a, h^{1,1}=b$, with $a \gg b$ ? Or say with $a$ arbitrarily large and $b=1$ or $b=2$ ? The same concrete question could be posed for threefolds, or more generally for the Hodge structures on $\mathrm{H}^{2}$ or $\mathrm{H}^{3}$. One can weaken the requirement by looking at motives. In this case, we just mean the image of an algebraically defined correspondence. Can the image Hodge structure have Hodge numbers with $a \gg b$ ? 
In formulating the same question for Betti numbers, care must be taken with the Lefschetz decomposition, which already provides the type of bound we are looking for in a certain sense. One could ask, do there exist varieties with large primitive cohomology $P^{i}$ and small $P^{j}$ for $i<j \leq(\operatorname{dim} X)$. Again here it might be convenient to distinguish odd and even cohomology.

When we see that we don't even know what to say about these reasonably simple questions, it is apparent that more complicated things such as the possible structure of the cohomology ring or just of certain fixed cup product operations, are completely open problems too. Without trying to list these questions exhaustively, we can formulate an example. If $X$ is a 3 -fold with a nontrivial vector space $V:=H^{2}(X, \mathrm{Q})$ then the cup product gives a cubic form $\eta \in\left(V^{*}\right)^{\otimes 3}$. What are the possibilities for the equivalence class of $(V, \eta)$ ? Even in the simplest case $\operatorname{dim} V=2$, the space of forms is 8-dimensional, and it is acted upon by the 4-dimensional group $G L(2)$; where does $\eta$ lie in the 4-dimensional space (or stack to be more precise) of orbits? What examples can we construct? The numerous possible generalizations of this type of question are perhaps better understood in terms of nonabelian cohomology.

The discussion wouldn't be complete without pointing out that the Hodge conjecture is an example (perhaps the first) of the construction problem. In that case, the problem is to construct subvarieties such that the cycle map gives a certain topological class. There are many different formulations, for example one can say that we want subvarieties such that the restriction of some cohomology class is nonzero. The situation for the Hodge conjecture remains much the same as the more general situation we are reflecting on here: we really don't have any good constructions of cycles. Indeed, most cases where it is known are obtained by showing that there aren't too many Hodge classes, in other words that there are sufficiently many restrictions on the classes that we don't have to look too far to get the rest. Two notable exceptions are Steenbrink's semi-regularity theorem [182] and Voisin's construction for Kummer surfaces in [194] (ii).

Another side of the story is related to our previous questions for Betti numbers. One of the simplest "standard conjectures" is algebraicity of the Kunneth projectors. But, even to construct varieties for which this question is interesting, we need ones with nontrivial Betti numbers in different degrees. It isn't even clear that we really know a very wide collection of such varieties

Let's move onto the non-simply connected case, and more specifically to questions about the fundamental group. One can note that by the existence of Artin neighborhoods, the topology of quasiprojective $K(\pi, 1)$ 's can be 
considered as the basic building block for the topology of any other varieties. (Also a recent result of Beilinson [23] and Katzarkov, Pantev and Toen [114] refines this to a statement about schematic homotopy types.) In this sense, it is natural to pay what might at first seem to be an inordinate amount of attention to questions about the fundamental group.

The situation is pretty well summed up by the first two sentences of Reznikov's paper [170] (vii):

Fundamental groups of complex projective varieties are very difficult to understand. There is a tremendous gap between few computed examples and few general theorems. $\cdots$

Reznikov is refering to what is actually a fairly diverse collection of constructions serving to give examples of fundamental groups. These range from uniformization constructions (which are also seen from other viewpoints e.g. in Deligne and Mostow [67], Livne [132]) to explicit constructions for example of nilpotent fundamental groups [44] [180]. See the discussion in the book of Amorós et al [7]. Nonetheless, this collection of examples leaves us with the impression that there are many examples of groups that we don't know about. It is not altogether trivial to formulate precise questions which express this feeling.

We can start with a very general one, which was inspired by Toledo's examples of varieties with non-residually finite $\pi_{1}$ [191] [7]. Can any finitely presented group be a subgroup of a $\pi_{1}(X)$ for a smooth projective variety $X$ ? Or, on the contrary, are there restrictions on which groups can occur as subgroups? As far as I know, none of the Hodge theory results that we currently have serves to rule out arbitrary subgroups. It is possible that some type of $L^{2}$ cohomology techniques, or maybe some Yang-Mills techniques such as in $\mathrm{Ni}$ and Ren [150], on the (possibly infinite) covering $\tilde{X} \rightarrow X$ determined by a subgroup $\Gamma \subset \pi_{1}(X)$, could give some restrictions. Toledo's example shows that one cannot hope for any results on this question using finite-dimensional Hodge theory.

On the construction side there is something obvious to try: given a finitely presented group $\Gamma$, it is the fundamental group of a smooth compact manifold $M$ (say, of dimension 4). This manifold can be embedded in some $\mathbf{R}^{N}$ and then approximated by a real algebraic manifold. Thus we may assume that $M$ is a connected component of the real points of an algebraic variety $X$ defined over $\mathbf{R}$. We can further replace $X$ by any covering whose ramification locus doesn't intersect $W$. We might hope in this way to get an injection $\pi_{1}(M) \hookrightarrow \pi_{1}(X)$. I don't see any way of proving that a map of this type is 
injective, though.

An interesting test case (suggested by M. Larsen) would be $\Gamma=G\left(n^{-1} \mathbf{Z}\right)$, the group of points in an algebraic group $G$ defined over $\mathbf{Z}$, with coefficients in a finite localization $n^{-1} \mathbf{Z}$. This group is linear, but cannot immediately be embedded in the fundamental group of a Hermitian symmetric domain quotient. One suspects that such $\Gamma$ cannot itself be a Kähler group, because it would tend to have rigid representations which are not integral, which would go against the conjecture that all rigid representations are motivic. It might, on the other hand, be possible to carry out a real-points construction such as in the previous paragraph, keeping enough control over a linear representation to prove injectivity of the map.

More generally, after Toledo's example and several others in the same direction (these are nicely presented in [7]), we can ask what type of nonresidually finite groups can occur. A folkloric observation/question ${ }^{9}$ is that in all examples so far, there is a nontrivial linear quotient group; do there exist Kähler groups without any subgroups of finite index at all?

A question which was recalled in E. Rees' Math Review MR1396672 of the paper by Amorós [4], as well as in [7] is whether there are groups which are fundamental groups of Kähler manifolds but not of smooth projective varieties. It might now be possible to give such an example, based on Voisin's construction [194].

One of the main invariants of a finitely presented group is its space of representations, say into $G L(n, \mathbf{C})$. It provides a way of measuring how well we can construct examples of fundamental groups. Say that a representation $\rho$ is non-rigid if its semisimplification lies in a positive-dimensional component of the moduli space of representations (recall that the moduli space parametrizes Jordan equivalence classes of representations, which is the same as isomorphism classes of semisimple representations). The nonrigidity condition stated this way implies nonvanishing of the deformation space, but the converse is not necessarily true: there can exist representations with nontrivial deformation space but where all the deformations are obstructed so there are no truely integrable deformations (and indeed this seems to be what happens in some Teichmüller examples, see below). The known factorization results leave ample room for existence of interesting nonrigid representations. However, the only examples I know are obtained in the following way: if $f: Z \rightarrow X$ is a morphism and if, for some reason we al-

\footnotetext{
${ }^{9}$ I don't remember who said it first but it wasn't me.
} 
ready have a nonrigid local system $V$ over $Z$, then some higher direct image $R^{i} f_{*} V$ will tend to be nonrigid on $X$. This construction has the notable property that the birational equivalence class of the irreducible component of the moduli space of representations of $\pi_{1}(X)$ which is constructed, is not necessarily any different from the one we started with for $\pi_{1}(Z)$. In other words, no new moduli spaces are constructed in this way, ${ }^{10}$ even if old moduli spaces are reinterpreted in new ways for new varieties.

This inevitably raises the question, do we already $\mathrm{know}^{11}$ all of the possible moduli spaces of representations? Or are there varieties having new moduli spaces not on the basic list? A first comment is that by the Lefschetz theorem restricting to a curve, any new moduli spaces are subspaces of moduli spaces of representations on a curve. This includes all of the structure, notably the hyperkähler structure [99] [78]. So one possible attack on this question would be to try to classify all hyperkähler subspaces of the moduli spaces of representations on curves. The extensive works of Verbitsky, Kaledin [193] [108] [109] are certainly relevant to any such project.

It is probably not easy to prove that two given hyperkähler varieties of the same dimension are distinct. For this part of the question, several possibilities might work: analysis of the singularities, looking at the power-series expansion of the hyperkähler structure at a special point, analysis of the structure at infinity, or calculation of the metric (e.g. curvature) properties. These things are all undoubtedly easier to do in low dimensions. This suggests that an important variant of the question would be, can we construct varieties whose space of representation has components with positive but low dimension? (Recall that the complex dimension is divisible by 2 , so the first possibilities would be dimension 2 or dimension 4.) Here we have fallen back into a situation analogous to that for the geography of surfaces: there are many natural examples of rigid representations, but for the "nearby" case of

\footnotetext{
${ }^{10}$ This is a bit of a misstatement. The moduli spaces are the same, if the collection of representations obtained as higher direct images from representations on $Z$ constitutes an irreducible component of the moduli space of representations on $X$. It of course remains possible that the representation on $X$ could acquire additional deformations in other directions not coming from deformations on $Z$. In this case we would get a candidate for a new moduli space (although that wouldn't be a proof that it was really different from the old ones, either ...). Comparing deformation spaces of the local system $V$ on $Z$ and the higher direct image $R^{i} f_{*} V$ on $X$ is therefore an interesting question.

${ }^{11}$ The known ones would be products of moduli spaces of representations on curves, and of rank one representations on abelian varieties.
} 
representations with low-dimensional moduli we know only very few ${ }^{12}$. For orbicurves (which also applies to certain fibrations with multiple fibers), the situation is much better: by work of Kostov [121] and Crawley-Boevey [55] we have a good classification of the irreducible components of the moduli spaces, and in particular there exist components of all possible (even) dimensions. Small components appeared in the "toy example" in [175].

At this juncture, note that cohomology support loci ${ }^{13}$ provide natural hyperkähler subvarieties. One might therefore ask whether the irreducible components of support loci occur as components of moduli spaces of representations. There might be geometric constructions relating the situation of a support locus, to a nonrigid representation. For these reasons (as well as because it fits into our general construction question) it would be interesting to have constructions of varieties giving rise to nontrivial cohomology support loci. As mentionned in the previous paragraph, a variant would be to ask for support loci of small positive dimension.

This brings us to a question raised by Hain and/or Looijenga (communicated to me by R.H. but he said it was more due to E.L.). They looked the moduli of $k$-pointed genus $g$ curves $M_{g, k}$ and looked at spaces of representations of $\pi_{1}\left(M_{g, k}\right)$ or perhaps more precisely the Teichmüller group which is the orbifold fundamental group. It is of course well-known that the standard representation is rigid. However, they found other representations (subobjects of tensor powers of the standard one) which were not infinitesimally rigid. The ones they found all had the property that there were enough obstructions that they were globally rigid even if not infinitesimally so. This then raises the question: are there any non-rigid (in our above sense) representations of $\pi_{1}\left(M_{g, k}\right)$ ? Or on the contrary are all representations globally rigid?

Actually if a variety $X$ has the property that all of its representations are globally rigid, then the pro-algebraic completion of $\pi_{1}(X)$ has a reasonable structure (something like a product of the relative Malcev completions at the various points) and similarly the schematic homotopy type $X \otimes \mathbf{C}$ as defined by Toen [189] [114] will have reasonable finiteness properties (for example the bad behavior noted at the beginning of Artin and Mazur [14] won't

\footnotetext{
${ }^{12}$ One should consider the possibility that this phenomenon might be substantial, rather than just a consequence of our lack of suitable constructions, see [53] (ii) for example.

${ }^{13}$ By "support loci" we mean the loci $\Sigma_{i, k}$ of local systems $V$ such that $\operatorname{dim}_{\mathbf{C}} H^{i}(X, V) \geq$ $k$. One could introduce variants here, such as loci where certain cup products in cohomology have a given rank.
} 
occur). This, plus the fact that the representations found so far by Hain and Looijenga turned out to be globally rigid in spite of their infinitesimal nonrigidity, leads us to conjecture that $\Gamma=\pi_{1}\left(M_{g, k}\right)$ doesn't have any globally non-rigid representations.

If the above questions aren't enough, one could pose similar ones for higher nonabelian cohomology [177]. The reader will readily imagine the details, so it doesn't seem necessary to stress this further here. The longrange hope is that nonabelian cohomology might help explain or organize what is going on-some hint of this can already be seen in the fact that it was natural to look at cohomology support loci.

We have been concentrating on the question of the pure topology of $X$. On the other hand, a great deal of research in differential geometry has looked at the classification of differential manifolds and other structures such as symplectic manifolds. A major part of this work has been to consider the place of algebraic or Kähler manifolds in the classification. Thus, what we know about the Donaldson-Seiberg-Gromov-Witten invariants of algebraic varieties, is quite comparable to what we know about them for other types of manifolds (I maintain that, as a whole, this is still not very much.) See [142], [77] [72] [154] for example. Lönne has considered an interesting question, which is the group of diffeomorphisms of a variety and its action on cohomology. He was able to calculate this in one case using Seiberg-Witten theory [133] (see also [103]). An important question is, to what extent will consideration of the new invariants help us for example to construct varieties with given Hodge numbers?

Another possible invariant that we could look to obtain would be intersection cohomology for singular varieties. All of the same types of questions could be posed in that context. One can also ask things like how many (and what kind of) singularities a variety can have, see [73] for example.

A general research direction, tangentially related to our present preoccupations, is the question of what happens when we go to "infinity". As became apparent with work of Wentworth and others, there are three natural directions to look at:

-We can go to infinity in an open (say, quasiprojective) variety, and ask for the behavior of representations, vector bundles, cohomology classes and so forth near the divisor at infinity.

-We can go to infinity in the space of representations. This leads to the whole subject of "singular perturbation theory", which is growing very diverse. There are lots of things to investigate. The question of how the 
harmonic metric degenerates when the representation goes to infinity was raised in [56] [58], in particular what relationship this has with harmonic maps to buildings occuring on the boundary of the moduli space of representations. There is also the whole question of the Stokes phenomenon for the asymptotic expansion of the monodromy, which actually is probably closely related to the harmonic map question, for example spectral curves play an important role in the Stokes phenomenon [61] [102] [9] [33].

-We can look at a degenerating family of varieties, considered as a smooth family over a punctured disc, and go to infinity at the puncture. This amounts to saying that we look at varieties where certain vanishing cycles become infinitely small. The degeneration of cohomology has been extensively studied in this case. The degeneration of the space of representations has been much less studied, see however [58] and [50] for example. Some time ago, Katzarkov raised the question of whether there is an analogue of the Clemens-Schmid exact sequence for spaces of representations or other nonabelian cohomology.

The above topics suggest their own formulations of the construction question. For example, do open varieties, or degenerating varieties, exist with given topological behavior? This is a vast question, and is also related to the smoothing and branching techniques for construction even of smooth projective examples.

Perhaps the biggest research area in Kähler geometry is the study of special metrics, for example Kähler-Einstein metrics or metrics with other rigidifying curvature properties [69] [186]. This continues to motivate a large segment of the field, and we couldn't go into sufficient detail here. This subject probably has or will have important implications for the topological construction problem we are discussing, on the one hand because the existence of metrics with special curvature properties implies strong topological restrictions, and on the other hand because this type of global analysis might contain key techniques for the construction of algebraic objects. A similar question is what new information will be brought by the Ricci flow techniques of Perelman [161]: can these be used to construct Hodge cycles by a minimization process, for example? More generally, is there a way in which one can look at "almost Kähler manifolds", then define obstructions and minimization processes allowing us to determine when an "almost Kähler manifold" could be deformed to a true one?

Now it's time to look at some of the main techniques for constructing varieties. The first and most obvious is by writing down equations. It is 
a nontrivial task just to understand what are the topological properties of a variety which is given by its equations. The other nontrivial task is to find systems of equations which don't reduce to complete intersections, ${ }^{14}$ a question which Landsberg has looked at in a theoretical way [123].

The equational method becomes very subtle already in codimension two. Perhaps a fundamental example is the determination by Gruson and Peskine of the values of $(d, g)$ for which there exist a smooth curve of degree $d$ and genus $g$ in $\mathbf{P}^{3}$ (which after all is an early version of the whole construction question we are considering). They succeeded by a very careful construction noticing that you got interesting examples out of the relatively large Picard groups of cubic and singular quartic surfaces. This type of method has not been sufficiently exploited in relationship with more overtly topological questions.

The next technique, which could sometimes be considered as a variant on the equational method, is to construct varieties as branched coverings of other varieties with a given branch locus. This has led to a large and intensive study of some of the first possible cases of branch loci, for example hyperplane arrangements, or curves with a few components of low degree in the projective plane [130] [97]. Fulton's theorem that $\pi_{1}$ is abelian if the branch curve has only nodes [80] shows that the question is subtle, see also [86]. The branched variety construction serves as a bridge between the topology of compact smooth varieties, and the topology of the open branch locus complements. In many cases it is easier to envision calculations, for example of fundamental groups, in the open case. This program has already been carried a long way by Moishezon and Teicher [140] with Robb [171] and others.

Another variant on the equational method is looking at degeneracy loci of vector bundle maps (see Decker, Ein, Schreyer who extensively investigated this in [60], see also [71]). Eyssidieux's construction of some varieties as base loci of linear systems [75] could also fit in here, although it has the additional subtlety that the linear system is $L^{2}$, taking place on an infinite covering - equivariance says that the base locus descends to a usual subvariety downstairs.

A hitherto underexplored idea is Kapovich and Millson's theorem saying

\footnotetext{
${ }^{14}$ Although it is not even completely clear that we fully understand the topology of complete intersections together with their embeddings, specially in twisted cases such as weighted projective spaces. A few references are [122], [131] but this is not a complete list.
} 
that any variety can be viewed as the moduli space of a linkage [110]. It is possible that the "mechanical" properties of a linkage could show up in the topological properties of the moduli space, and in this way we might have a more natural relationship between the presentation of a variety and its topology. It seems urgent to look at this in more detail.

A generalization would be to try to construct varieties as moduli spaces of any kind of geometric object, and to hope that the topological properties of the moduli spaces would be related to the moduli problem. This is of course what happens with well-known moduli spaces. However, up to now the philosophical take on this theory has always been that the "moduli spaces" are natural objects, and we should be interested in their topological properties. A more intentional approach would say that we are interested in constructing given topological types, and we would like to pick and choose our moduli problem in order to get what we want. One place to start might be to look at moduli spaces of group representations (a point of view present in [110]). For strange groups $\Gamma$ which the researcher should carefully choose (probably not Kähler ones!), does the moduli space of representations of $\Gamma$ have an interesting topological type? Other possibilities might include components of the moduli spaces of space curves [91], or (related by [16]) components of the Noether-Lefschetz loci [48] [155].

Something which was mentionned previously is the existence of uniformization constructions for algebraic varieties. The simplest examples are direct construction of the quotient of a symmetric domain by a discrete group action. In recent years this has been considerably extended. Perhaps one of the first extensions was Toledo's construction of examples with non-residually finite fundamental group [191]. The idea is that certain natural subspaces of symmetric domains can fit together into interesting configurations in the quotient variety. Then other techniques such as branched covers, or in Toledo's case blowing down a negative subvariety and then taking hyperplane sections which miss the singularities, give new varieties. Lately some more complicated configurations have been considered by Allcock, Carlson and Toledo [3]. These types of constructions are good candidates; one of the major difficulties is in calculating what happens.

Another important technique is the notion of fibration, which came up under the notion of Lefschetz devissage above. Whereas one often uses fibrations to study the topology of a variety, it is also possible to try to construct varieties by explicitly constructing a fibration. The easiest example is that of Kodaira surfaces, where the fibration is automatically smooth (a construction 
which easily generalizes in higher dimensions too). Recall that the basic idea consists of saying that given a pointed curve we can take a covering ramified at the point. As the point moves, this gives a family of curves fibering over the original curve; the construction can be iterated in the fibers. One can write down the group automorphisms determining the monodromy, but the formulae tend to be long and not very enlightening. Things might get more tractable by putting computers to work to do the calculations. Other examples of fibration constructions include the ones of Bogomolov and Katzarkov [35] - but here again it is difficult to know what the result is. This theory of course spills over into the symplectic world via Donaldson's notion of almostLefschetz fibration. Gompf [82], Amorós et al [6], Reznikov [170] (ii) show that the symplectic "almost-Lefschetz" world is more flexible in an essential way, for example any fundamental group can arise. In view of these results, it doesn't seem clear what implications there are for our present construction problem.

Braid groups come into play when looking at fibrations whose fibers are punctured projective lines [140]. In this case, a family is just a moving system of points in $\mathbf{P}^{1}$. The de Rham version of the devissage for representations of the fundamental group is what is often known as "isomonodromic deformation", or "Painlevé equations". The isomonodromy equations or nonabelian Gauss-Manin, are equations which tell us when we have a family of representations on the fibers which fit together to give a representation of the fundamental group of the total space. So, looking for algebraic solutions of these equations could be a way of constructing quasiprojective varieties with nonrigid representations. Some solutions have been found by Boalch [33] and Hitchin [99].

Birational transformations enter into algebraic geometry in many ways, and they constitute a way of modifying a variety which changes its topological type in a readily calculable way. These provide a tool for transforming a subvariety into a modification, for example. Also a singularity could become a topological feature after resolution. This type of construction showed up very successfully in Voisin's examples of compact Kähler varieties whose homotopy types cannot be smooth projective [194].

One of the major potential techniques for construction of smooth projective varieties is smoothing of degenerate configurations. This was mentionned, for example, in Peters' "Math Review" [166] of U. Persson's paper [162]. This tool plays an important role in the determination of the "geography of surfaces" [163]. While not immediately trivial, the calculation of 
the homotopy type of the smooth nearby fiber is something which can be done reasonably well using traditional Hodge-theoretic techniques. One of the main difficulties is that not enough research has been done to establish useful criteria stating when a configuration can be smoothed. This has to be a subtle problem [165], because all of the Hodge-theoretic restrictions on homotopy types show that you can't just smooth any configuration. Smoothings are known to exist in some special cases [90] [168] [169] [186] [188]. There is a wide choice of initial configuration, which could be a union of projective spaces or other easy pieces - in which case the combinatorics of the configuration would determine the topology of the nearby fiber; or it could on the other hand be a variety having a complicated singularity which contributes a lot of topology in the smoothing.

Whatever methods are used for constructing varieties, there is always the problem of calculating what you get. The calculation problem is rendered difficult by the fact that, not only do we need to know how to find the answer, but we need a sufficiently agile understanding of how the calculation works that we can choose the construction accordingly. Indeed, the main point of the whole "construction question" we are asking here is to be able to influence the output topological type as much as possible, by varying the input construction of the variety. ${ }^{15}$

Some recent advances in Hodge theory are likely to be key elements of this computational aspect. Notable is T. Mochizuki's work on nonabelian Hodge theory on open varieties [138], see previously [32] [128]. We will describe in a bit more detail how this is relevant. In the short range, one of the most promising avenues for constructing things remains the consideration of complements of configurations of curves in $\mathbf{P}^{2}$ (maybe one would blow up at singular points to obtain a normal crossings configuration). The isomonodromy equations constitute one approach to look for representations of the fundamental group of the complement. However, a more global approach would be to look for harmonic bundles over $\mathbf{P}^{2}$ with singularities along the curves in the configuration. There are three incarnations we could use to look for these: the Dolbeault incarnation would say to look for logarithmic parabolic Higgs bundles; the de Rham incarnation, to look for parabolic bundles with logarithmic connexion; and the Betti incarnation (hitherto less explored) would be to look for "filtered local systems". T. Mochizuki's work describes the properties of harmonic bundles on open varieties and their ex-

\footnotetext{
${ }^{15}$ Some kind of "engineering" in the wonderful spirit of the title of [25].
} 
tensions to the normal crossings compactification. There is still a lot to be done here, but he has crossed the essential step of understanding the local behavior at the singularities. Thus, we can imagine that in the semi-near future we will have the full panoply of tools necessary to analyze the correspondence between the Dolbeault, de Rham and Betti points of view. In each case, there should be a Bogomolov-Gieseker inequality (for the logarithmic Dolbeault case this was mentionned to me recently by Narasimhan). It is the limit case of this inequality which gives rise to representations. The case of filtered local systems is worth highlighting. The easiest possibility would be to look at filtrations on the trivial local system. Then a filtered structure is just specification of a filtration (indexed by real weights) for each divisor in the configuration. The parabolic Chern classes would be polynomials in the weights whose coefficients are determined by the combinatorics of which divisors intersect. The Bogomolov-Gieseker inequality might well give nontrivial constraints on the possible combinatorics of the configuration; and when the limiting case of equality is attained, one would get families of harmonic bundles which, after transformations such as described in [177] (which would require the fully worked-out theory to implement), would give representations of the fundamental group on the complement. It would then become a combinatorial problem to find configurations where the limiting case of equality could be attained. We can vividly hope that T. Mochizuki's work will allow us to carry out this type of investigation.

It is really unclear what type of answer we would expect from calculations such as described in the previous paragraph. We would be looking to attain the limiting case of a Bogomolov-Gieseker type inequality, and that would seem hard to do. A lot of negative results formalizing this difficulty would lend credence to the conjecture on Teichmüller groups stated above, and would also increase the hope for some type of strong classification results about representations. Generalizing the rank two classification of [54], it might be possible to get say a classification for rank three representations.

If there is any conclusion to be drawn, it is perhaps that even though there is no single fully satisfactory choice of a method of construction, nonetheless there are many methods which haven't been fully exhausted yet; and similarly there are so many different variants of the basic construction question, that maybe we should just spend some time trying to see what topological shapes can be constructed with the methods currently at hand. This might direct us towards the most promising construction methods, and might also lead to ideas for improving the underlying Hodge-theoretical tools of calculation. 


\section{References}

[1] D. Abramovich, A.J. de Jong. Smoothness, semistability, and toroidal geometry. J. Algebraic Geom. 6 (1997), 789-801.

[2] E. Allaud. Variation de structure de Hodge et systèmes différenteils extérieurs. Thesis, Université Paul Sabatier (2002). See http://fermat.ups-tlse.fr/ webthesards/theses.htm.

[3] D. Allcock, J. Carlson, D. Toledo. Orthogonal complex hyperplane arrangements. Symposium in Honor of C. H. Clemens (Salt Lake City 2000), Contemp. Math. 312 (2002), 1-8.

[4] J. Amorós. On the Malcev completion of Kähler groups. Comment. Math. Helv. 71 (1996), 192-212.

[5] J. Amorós, I. Bauer. On the number of defining relations for nonfibered Kähler groups. Internat. J. Math. 11 (2000), 285-290.

[6] J. Amorós, F. Bogomolov, L. Katzarkov, T. Pantev. Symplectic Lefschetz fibrations with arbitrary fundamental groups. Appendix by Ivan Smith. J. Diff. Geom. 54 (2000), 489-545.

[7] J. Amorós, M. Burger, K. Corlette, D. Kotschick, D. Toledo. Fundamental groups of compact Kähler manifolds. A.M.S. MATHEMATICAL Surveys And Monographs, 44 (1996).

[8] Y. André. (i) Pour une théorie inconditionnelle des motifs. Publ. Math. I.H.E.S. 83, (1996), 5-49.

(ii) Quelques conjectures de transcendence issues de la géométrie algébrique. Preprint Institut de Mathématiques de Jussieu 121 (1997).

[9] T. Aoki, T. Kawai, Y. Takei. On the exact steepest descent method: A new method for the description of Stokes curves. J. Math. Phys. 42 (2001), 3691-3713.

[10] V. Apostolov, O. Muskarov. Weakly Einstein-Hermitian surfaces. Ann. Inst. Fourier 49 (1999), 1673-1692.

[11] D. Arapura. (i) Hodge theory with local coefficients and fundamental groups of varieties. Bull. Amer. Math. Soc. 20 (1989), 169-172. 
(ii) Smoothable varieties with torsion free canonical sheaf. Manuscripta Math. 63 (1989), 17-20.

(iii) Fundamental groups of smooth projective varieties. Current topics in complex algebraic geometry (Berkeley, 1992/93), MSRI PUBL. 28, Cambridge Univ. Press (1995), 1-16.

(iv) Geometry of cohomology support loci for local systems, I. J. Algebraic Geom. 6 (1997), 563-597.

[12] D. Arapura, P. Bressler, M. Ramachandran. On the fundamental group of a compact Kähler manifold. Duke Math. J. 68 (1992), 477-488.

[13] D. Arapura, M. Nori. Solvable fundamental groups of algebraic varieties and Kähler manifolds. Compositio Math. 116 (1999), 173-188.

[14] M. Artin, B. Mazur. Etale homotopy. Springer L.N.M. 100 (1969).

[15] D. Auroux, L. Katzarkov. Branched coverings of $\mathbf{C P}^{2}$ and invariants of symplectic 4-manifolds. Inventiones 142 (2000), 631-673.

[16] S. Azziz. Exemples de composantes irréductibles et non réduites du schéma de Hilbert des courbes lisses et connexes de $\mathbf{P}^{3}$ (II). Preprint alg-geom/9701018.

[17] V. Balaji, I. Biswas, D. Nagaraj. Principal bundles over projective manifolds with parabolic structure over a divisor. Tohoku Math. J. 53 (2001), 337-367.

[18] S. Bando, Y.-T. Siu. Stable sheaves and Einstein-Hermitian metrics. Geometry and analysis on complex manifolds, World Sci. Publishing (1994), 39-50.

[19] I. Bauer. Irrational pencils on non-compact algebraic manifolds. Internat. J. Math. 8 (1997), 441-450.

[20] T. Bauer. Seshadri constants and periods of polarized abelian varieties. (appendix by the author and T. Szemberg) Math. Ann. 312 (1998), 607-623.

[21] I. Bauer, F. Catanese. Some new surfaces with $p_{g}=q=0$. Preprint math. AG/0310150, to appear in the Proceedings of the Fano Conference (Torino, 2002). 
[22] A. Beauville. A Calabi-Yau threefold with non-abelian fundamental group. New trends in algebraic geometry (Warwick, 1996) LONDON Math. Soc. Lecture Note Ser. 264 Cambridge Univ. Press (1999), 13-17.

(ii) Quantum cohomology of complete intersections. Mat. Fiz. Anal. Geom. 2 (1995), 384-398.

(iii) Varits Kähleriennes dont la première classe de Chern est nulle. J. Diff. Geom. 18 (1983), 755-782.

[23] A. Beilinson. (i) Higher regulators and values of $L$-functions. Current problems in mathematics 24 Itogi Nauki i Tekhniki, Akad. Nauk SSSR, Vsesoyuz. Inst. Nauchn. I Tekhn. Inform., Moscow (1984), 181-238.

(ii) Notes on absolute Hodge cohomology. Applications of algebraic $K$ theory to algebraic geometry and number theory (Boulder, 1983) CoNTEMP. MATH. 55, AMS (1986), 35-68.

(iii) On the derived category of perverse sheaves. K-theory, arithmetic and geometry (Moscow, 1984-1986), Springer L.N.M. 1289 (1987), 2741.

[24] E. Bierstone, P. Milman. Canonical desingularization in characteristic zero by blowing up the maximum strata of a local invariant. Invent. Math. 128 (1997), 207-302.

[25] M. Bershadsky, A. Johansen, T. Pantev, V. Sadov, C. Vafa. F-theory, geometric engineering and $N=1$ dualities. Nuclear Phys. B 505 (1997), 153-164.

[26] A. Berthomieu. Proof of Nadel's conjecture and direct image for relative K-theory. Bull. S.M.F. 130 (2002), 253-307.

[27] J. Bertin, J.-P. Demailly, L. Illusie, C. Peters. Introduction à la théorie de Hodge. Panoramas et Synthèses 3, S.M.F., Paris (1996).

[28] F. Bihan. Asymptotiques de nombres de Betti d'hypersurfaces projectives réelles. Preprint math. AG/0312259.

[29] I. Biswas. (i) Vector bundles with holomorphic connection over a projective manifold with tangent bundle of nonnegative degree. Proc. Amer. Math. Soc. 126 (1998), 2827-2834. 
(ii) Flat connections on a punctured sphere and geodesic polygons in a Lie group. J. Geom. Phys. 39 (2001), 129-134.

[30] I. Biswas, T. Gómez. A Torelli theorem for the moduli space of Higgs bundles on a curve. Q. J. Math. 54 (2003), 159-169.

[31] I. Biswas, M. Mitra, S. Nag. Thurston boundary of Teichmller spaces and the commensurability modular group. Conform. Geom. Dyn. 3 (1999), $50-66$.

[32] O. Biquard. Sur les fibrés paraboliques sur une surface complexe. J. London Math. Soc. 53 (1996), 302-316.

[33] P. Boalch. The Klein solution to Painleve's sixth equation. Preprint math. AG/0308221.

[34] H. Boden, K. Yokogawa. Moduli spaces of parabolic Higgs bundles and parabolic $K(D)$ pairs over smooth curves, I. Internat. J. Math. 7 (1996), 573-598.

[35] F. Bogomolov, L. Katzarkov. Complex projective surfaces and infinite groups. Geom. Funct. Anal. 8 (1998), 243-272.

[36] F. Bogomolov, T. Pantev. Weak Hironaka theorem. Math. Res. Lett. 3 (1996), 299-307.

[37] S. Bradlow. Special metrics and stability for holomorphic bundles with global sections. J. Differential Geom. 33 (1991), 169-214.

[38] S. Bradlow, G. Daskalopolous. Moduli of stable pairs for holomorphic bundles over Riemann surfaces, II. Int. J. Math 4 (1993), 903-925.

[39] S. Bradlow, G. Daskalopolous, R. Wentworth. Birational equivalence of vortex moduli. Topology 35 (1996), 731-748.

[40] S. Bradlow, O. García-Prada, P. Gothen. Surface group representations and $U(p, q)$-Higgs bundles. J. Diff. Geom. 64 (2003), 111-170.

[41] S. Broughton. On the topology of polynomial hypersurfaces. Singularities, Part 1 (Arcata, Calif., 1981) AMS Proc. Sympos. Pure Math. 40 (1983), 167-178, 
[42] P. Buser, P. Sarnak. On the period matrix of a Riemann surface of large genus. (appendix by J. Conway and N. Sloane). Inventiones 117 (1994), $27-56$.

[43] E. Calabi, X. Chen. The space of Kähler metrics. II. J. Differential Geom. 61 (2002), 173-193.

[44] F. Campana. (i) Remarques sur les groupes de Kähler nilpotents. Ann. Sci. E.N.S. 28 (1995), 307-316.

(ii) Ensembles de Green-Lazarsfeld et quotients résolubles des groupes de Kähler. J. Algebraic Geom. 10 (2001), 599-622.

(iii) Connexité abélienne des variétés kählériennes compactes. Bull. S.M.F. 126 (1998), 483-506.

[45] J. Carlson, D. Toledo. (i) Harmonic mappings of Kähler manifolds to locally symmetric spaces. Publ. Math. I.H.E.S. 69 (1989), 173-201.

(ii) Rigidity of harmonic maps of maximum rank. J. Geom. Anal. 3 (1993), 99-140.

(iii) Quadratic presentations and nilpotent Kähler groups. J. Geom. Anal. 5 (1995), 359-377.

(iv) On fundamental groups of class VII surfaces. Bull. London Math. Soc. 29 (1997), 98-102.

(v) Discriminant complements and kernels of monodromy representations. Duke Math. J. 97 (1999), 621-648.

[46] J. Carlson, H. Clemens, J. Morgan. On the mixed Hodge structure associated to $\pi_{3}$ of a simply connected complex projective manifold. Ann. Sci. E.N.S. 14 (1981), 323-338.

[47] F. Catanese. (i) Moduli and classification of irregular Kähler manifolds (and algebraic varieties) with Albanese general type fibrations, Inventiones 104 (1991), 263-289 (Appendix by A. Beauville).

(ii) Fundamental groups with few relations. Higher Dimensional Complex Varieties, Trento 1994. De Gruyter (1996), 163-165.

[48] E. Cattani, P. Deligne, A. Kaplan. On the locus of Hodge classes. J. Amer. Math. Soc. 8 (1995), 483-506.

[49] E. Cattani, A. Kaplan, W. Schmid. (i) $L^{2}$ and intersection cohomologies for a polarizable variation of Hodge structure. Inventiones 87 (1987), 
$217-252$.

(ii) Degeneration of Hodge structures. Ann. of Math. 123 (1986), 457535 .

[50] J. Chen, G. Tian. Compactification of moduli space of harmonic mappings. Comment. Math. Helv. 74 (1999), 201-237.

[51] H. Clemens. (i) Degeneration techniques in the study of threefolds. Algebraic threefolds, Varenna, 1981, Springer L.N.M. 947 (1982), 93-154. (ii) Degeneration of Kähler manifolds. Duke Math. J. 44 (1977), 215290.

[52] D. Cohen, P. Orlik. Arrangements and local systems. Math. Res. Lett. 7 (2000), 299-316.

[53] K. Corlette. (i) Flat G-bundles with canonical metrics. J. Diff. Geom. 28 (1988), 361-382.

(ii) Rigid representations of Kählerian fundamental groups. J. Diff. Geom. 33 (1991), 239-252.

(iii) Nonabelian Hodge theory. Differential geometry: geometry in mathematical physics and related topics (Los Angeles, 1990), AMS Proc. Sympos. Pure Math. 54 (1993), 125-144.

(iv) Nonabelian Hodge theory and square integrability. Actes du colloque Géométrie et Topologie des Variétés Projectives, Toulouse 1992, Preprint Toulouse (1992).

[54] K. Corlette, C. Simpson. The classification of rank two local systems on algebraic varieties. A paper that we are still planning to write.

[55] W. Crawley-Boevey. On matrices in prescribed conjugacy classes with no common invariant subspace and sum zero. Duke Math. J. 118 (2003), 339-352.

[56] G. Daskalopolous, S. Dostoglou, R. Wentworth. (i) On the MorganShalen compactification of the $S L(2, \mathbf{C})$ character varieties of surface groups. Duke Math. J. 101 (2000), 189-207.

(ii) Character varieties and harmonic maps to $R$-trees. Math. Res. Lett. 5 (1998), 523-533.

[57] G. Daskalopolous, L. Katzarkov, R. Wentworth. Harmonic maps to Teichmüller space. Math. Res. Lett. 7 (2000), 133-146. 
[58] G. Daskalopoulos, R. Wentworth. (i) The Yang-Mills flow near the boundary of Teichmüller space. Math. Ann. 318 (2000), 1-42.

(ii) Local degeneration of the moduli space of vector bundles and factorization of rank two theta functions I. Math. Ann. 297 (1993), 417-466.

[59] B. de Oliveira, L. Katzarkov, M. Ramachandran. Deformations of large fundamental groups. Geom. Funct. Anal. 12 (2002), 651-668.

[60] W. Decker, L. Ein, F. Schreyer. Construction of surfaces in $\mathbf{P}^{4}$. J. Algebraic Geom. 2 (1993), 185-237.

[61] E. Delabaere, H. Dillinger, F. Pham. Résurgence de Voros et périodes des courbes hyperelliptiques. Ann. Inst. Fourier 43 (1993), 163-199.

[62] E. Delabaere, J. Howls. Global asymptotics for multiple integrals with boundaries. Duke Math. J. 112 (2002), 199-264.

[63] P. Deligne. (i) Théorie de Hodge II. Publ. Math. IHES 40 (1971), 5-58.

(ii) La conjecture de Weil I. Publ. Math. IHES 43 (1974), 273-307.

(iii) Théorie de Hodge III. Publ. Math. IHES 44 (1974), 5-77.

[64] P. Deligne, A. Dimca. Filtrations de Hodge et par l'ordre du pôle pour les hypersurfaces singulières. Ann. Sci. E.N.S. 23 (1990), 645-656.

[65] P. Deligne, P. Griffiths, J. Morgan, D. Sullivan. Real homotopy theory of Kähler manifolds. Inventiones 29 (1975), 245-274.

[66] P. Deligne, J. Milne, A. Ogus, K. Shih. Hodge cycles, motives, and Shimura varieties. Springer L.N.M. 900 (1982).

[67] P. Deligne, G. Mostow. (i) Monodromy of hypergeometric functions and nonlattice integral monodromy. Publ. Math. I.H.E.S. 63 (1986), 5-89.

(ii) Commensurabilities among lattices in PU $(1, n)$. Ann. OF Math. Studies 132, Princeton University Press (1993).

[68] A. Dimca, A. Nemethi. On the monodromy of complex polynomials. Duke Math. J. 108 (2001), 199-209.

[69] S. Donaldson. (i) Symplectic submanifolds and almost-complex geometry. J. Diff. Geom. 44 (1996), 666-705.

(ii) Scalar curvature and projective embeddings. I. J. Diff. Geom. 59 
(2001), 479-522.

(iii) Planck's constant in complex and almost-complex geometry. XIIIth International Congress on Mathematical Physics (London, 2000), Int. Press, (2001), 63-72.

(iv) Infinite determinants, stable bundles and curvature. Duke Math. J. 54 (1987), 231-247.

(v) Anti self-dual Yang-Mills connections over complex algebraic surfaces and stable vector bundles. Proc. London Math. Soc. 50 (1985), $1-26$.

[70] L. Ein, R. Lazarsfeld. (i) Seshadri constants on smooth surfaces. Journes de Géométrie Algébrique d'Orsay, 1992, AstéRISQue 218 (1993), 177186.

(ii) Singularities of theta divisors and the birational geometry of irregular varieties. J. Amer. Math. Soc. 10 (1997), 243-258.

[71] D. Eisenbud, S. Popescu, C. Walter. Lagrangian subbundles and codimension 3 subcanonical subschemes. Duke Math. J. 107 (2001), 427-467.

[72] G. Ellingsrud, J. Le Potier, S. A. Stromme. Some Donaldson invariants of $\mathbf{C P}^{2}$. Moduli of vector bundles (Sanda, Kyoto, 1994) LECT. NoteS Pure Appl. Math. 179 (1996), 33-38.

[73] S. Endrass, U. Persson, J. Stevens. Surfaces with triple points. J. Algebraic Geom. 12 (2003), 367-404.

[74] H. Esnault, M. Nori, V. Srinivas. Hodge type of projective varieties of low degree. Math. Ann. 293 (1992), 1-6.

[75] P. Eyssidieux. (i) La caractéristique d'Euler du complexe de GaussManin. J. Reine Angew. Math. 490 (1997), 155-212.

(ii) Un théoréme de Nakai-Moishezon pour certaines classes de type $(1,1)$. Preprint math. AG/9811065.

(iii) Invariants de Von Neumann des faisceaux coherents. Preprint math. AG/9806159.

(iv) Sur la convexité holomorphe des revêtements lináires réductifs d'une variété projective algébrique complexe. Prépublication 179, Laboratoire E. Picard, Toulouse (2000). To appear.

[76] B. Feix. Hyperkähler metrics on cotangent bundles. J. Reine Angew. Math. 532 (2001), 33-46. 
[77] R. Friedman, J. Morgan. Algebraic surfaces and Seiberg-Witten invariants. J. Algebraic Geom. 6 (1997), 445-479.

[78] A. Fujiki. (i) An $L^{2}$ Dolbeault lemma and its applications. Publ. R.I.M.S. 28 (1992), 845-884.

(ii) Hyper-Kähler structure on the moduli space of flat bundles. Prospects in complex geometry (Katata and Kyoto, 1989), Springer L.N.M. 1468 (1991), 1-83.

[79] A. Fujiki, R. Kobayashi, S. Lu. On the fundamental group of certain open normal surfaces. Saitama Math. J. 11 (1993), 15-20.

[80] W. Fulton, On the fundamental group of the complement of a node curve. Ann. of Math. 111 (1980), 407-409.

[81] W. Goldman, J. Millson. The deformation theory of representations of fundamental groups of compact Kähler manifolds. Publ. Math. I.H.E.S. 67 (1988), 43-96.

[82] R. Gompf. A new construction of symplectic manifolds. Ann. of Math. 142 (1995), 527-595.

[83] R. Gompf, T. Mrowka. Irreducible 4-manifolds need not be complex. Ann. of Math. 138 (1993), 61-111.

[84] P. Gothen. Components of spaces of representations and stable triples. Topology 40 (2001), 823-850.

[85] M. Green, R. Lazarsfeld. Higher obstructions to deforming cohomology groups of line bundles. J. Amer. Math. Soc. 4 (1991), 87-103.

[86] G.-M. Greuel, C. Lossen, E. Shustin. Plane curves of minimal degree with prescribed singularities. Inventiones 133 (1998), 539-580.

[87] P. Griffiths, J. Harris. Principles of Algebraic Geometry. WileyInterscience, New York (1978).

[88] M. Gromov. Sur le groupe fondamental d'une variété kählérienne, C.R.A.S. 308 (1989), 67-70. 
[89] M. Gromov, R. Schoen. Harmonic maps into singular spaces and p-adic superrigidity for lattices in groups of rank one. Publ. Math. I.H.E.S. 76 (1992), 165-246.

[90] M. Gross. Deforming Calabi-Yau threefolds. Math. Ann. 308 (1997), 187-220. See also the references mentionned in the Math Reviews MR1464900 (98i:14039) of this paper.

(ii) The deformation space of Calabi-Yau $n$-folds with canonical singularities can be obstructed. Mirror symmetry, II AMS/IP STUd. ADV. MATH. 1 (1997), 401-411.

[91] L. Gruson, C. Peskine. Genre des courbes de l'espace projectif. Algebraic geometry (Tromso, 1977), Springer L.N.M. 687 (1978), 31-59.

[92] D. Guan. On the Betti numbers of irreducible compact hyperkähler manifolds of complex dimension four. Math. Res. Lett. 8 (2001), 663-669.

[93] R. Hain. (i) The de Rham homotopy theory of complex algebraic varieties, I. K-Theory 1 (1987), 271-324.

(ii) The de Rham homotopy theory of complex algebraic varieties. II. K-Theory 1 (1987), 481-497.

(iii) Infinitesimal presentations of the Torelli groups. J. Amer. Math. Soc. 10 (1997), 597-651.

(iv) The Hodge de Rham theory of relative Malcev completion. Ann. Sci. E.N.S. 31 (1998), 47-92.

(v) The rational cohomology ring of the moduli space of abelian 3-folds. Math. Res. Lett. 9 (2002), 473-491.

[94] R. Hain, E. Looijenga. Mapping class groups and moduli spaces of curves. Algebraic geometry, Santa Cruz 1995, A.M.S. Proc. Sympos. Pure Math. 62 (2) (1997), 97-142.

[95] T. Hausel. Compactification of moduli of Higgs bundles. J. Reine Angew. Math. 503 (1998), 169-192.

[96] T. Hausel, M. Thaddeus. Mirror symmetry, Langlands duality, and the Hitchin system. Inventiones 153 (2003), 197-229.

[97] E. Hironaka. Alexander stratifications of character varieties. Ann. Inst. Fourier 47 (1997), 555-583. 
[98] H. Hironaka. Resolution of singularities of an algebraic variety over a field of characteristic zero. I, II. Ann. of Math. 79 (1964), 109-203; 205326.

[99] N. Hitchin. (i) Stable bundles and integrable systems. Duke Math. J. 54 (1987), 91-114.

(ii) The self-duality equations on a Riemann surface. Proc. London Math. Soc. 55 (1987), 59-126.

(iii) Poncelet polygons and the Painlevé equations. Geometry and analysis (Bombay, 1992), Tata Inst. Fund. Res., Bombay (1995), 151-185.

[100] B. Ilic, J.M. Landsberg. On symmetric degeneracy loci, spaces of symmetric matrices of constant rank and dual varieties. Math. Ann. 314 (1999), 159-174.

[101] Y. Imayoshi, M. Ito, Manabu; H. Yamamoto. On the Nielsen-ThurstonBers type of some self-maps of Riemann surfaces with two specified points. Osaka J. Math. 40 (2003), 659-685.

[102] M. Inaba, K. Iwasaki, Masa-Hiko Saito. Moduli of Stable Parabolic Connections, Riemann-Hilbert correspondence and Geometry of Painlevé equation of type VI, Part I. Preprint math.AG/0309342.

[103] D. Joe, M. Kim. On the topology of algebraic surfaces and reduction modulo p. Int. Math. Res. Not. 28 (2002), 1505-1508.

[104] F. Johnson, E. Rees. On the fundamental group of a complex algebraic manifold. Bull. London Math. Soc. 19 (1987), 463-466.

[105] J. Jost, K. Zuo. (i) Arakelov type inequalities for Hodge bundles over algebraic varieties, I. Hodge bundles over algebraic curves. J. Algebraic Geom. 11 (2002), 535-546.

(ii) Representations of fundamental groups of algebraic manifolds and their restrictions to fibers of a fibration. Math. Res. Lett. 8 (2001), 569575 .

(iii) Vanishing theorems for $L^{2}$-cohomology on infinite coverings of compact Kähler manifolds and applications in algebraic geometry. Comm. Anal. Geom. 8 (2000), 1-30.

(iv) Harmonic maps into Bruhat-Tits buildings and factorizations of $p$-adically unbounded representations of $\pi_{1}$ of algebraic varieties, I. J. 
Algebraic Geom. 9 (2000), 1-42.

(v) Harmonic maps of infinite energy and rigidity results for representations of fundamental groups of quasiprojective varieties. J. Diff. Geom. 47 (1997), 469-503.

(vi) Harmonic maps and $S L(r, \mathbf{C})$-representations of fundamental groups of quasiprojective manifolds. J. Algebraic Geom. 5 (1996), $77-106$.

[106] J. Jost, Y. Yang, K. Zuo. The cohomology of a variation of polarized Hodge structures over a quasi-compact Kähler manifold. Preprint math. AG/0312145.

[107] A. Kabanov. The second cohomology with symplectic coefficients of the moduli space of smooth projective curves. Compositio Math. 110 (1998), 163-186.

[108] D. Kaledin. Hyperkaehler structures on total spaces of holomorphic cotangent bundles. Preprint alg-geom/9710026.

[109] D. Kaledin, M. Verbitsky. (i) Hyperkähler manifolds. MathematicAL PHysics 12, International Press (1999).

(ii) Period map for non-compact holomorphically symplectic manifolds. Geom. Funct. Anal. 12 (2002), 1265-1295.

[110] M. Kapovich, J. Millson. (i) On representation varieties of Artin groups, projective arrangements and the fundamental groups of smooth projective varieties. Publ. Math. I.H.E.S. 88 (1998), 5-95.

(ii) Universality theorems for configuration spaces of planar linkages. Topology 41 (2002), 1051-1107.

(iii) Hodge theory and the art of paper folding. Publ.R.I.M.S. 33 (1997), $1-31$.

[111] L. Katzarkov. (i) Schematization of homotopy types and realizations. Comm. in arithmetic fundamental groups, Kyoto 1999-2001 KURAKAISEKIKENKYUSHO KOKYUROKU 1267 (2002), 147-156.

(ii) On the Shafarevich maps. Algebraic geometry, Santa Cruz 1995 AMS Proc. Symp. Pure Math. 62 (2) (1997), 173-216.

(iii) The geometry of large fundamental groups. J. Math. Sci. 94 (1999), 1100-1110. 
[112] L. Katzarkov, T. Pantev. (i) Representations of fundamental groups whose Higgs bundles are pullbacks. J. Differential Geom. 39 (1994), 103121.

(ii) Nonabelian $(p, p)$-classes. Motives, polylogarithms and Hodge theory, Irvine, 1998 Int. Press Lecture Ser. 3 (II) (2002), 625-715.

[113] L. Katzarkov, T. Pantev, C. Simpson. (i) Density of monodromy actions on non-abelian cohomology. Adv in Math. 179 (2003), 155-204

(ii) Nonabelian mixed Hodge structures. Preprint math. AG/0006213.

[114] L. Katzarkov, T. Pantev, B. Toen. Schematic homotopy types and non-abelian Hodge theory I: The Hodge decomposition. Preprint math. AG/0107129.

[115] L. Katzarkov, M. Ramachandran. On the universal coverings of algebraic surfaces. Ann. Sci. E.N.S. 31 (1998), 525-535.

[116] M. Kim, A. Sommese. Two results on branched coverings of Grassmannians. J. Math. Kyoto Univ. 38 (1998), 21-27.

[117] B. Klingler. (i) Structures affines et projectives sur les surfaces complexes. Ann. Inst. Fourier 48 (1998), 441-477.

(ii) Sur la rigidité de certains groupes fondamentaux, l'arithméticité des réseaux hyperboliques complexes, et les "faux plans projectifs". Inventiones 153 (2003), 105-143.

[118] H. Konno. Construction of the moduli space of stable parabolic Higgs bundles on a Riemann surface. J. Math. Soc. Japan 45 (1993), 253-276.

[119] M. Kontsevich, Y. Manin, Gromov-Wiiten classes, quantum cohomology, and enumeration geometry. Comm. Math. Phys. 164 (1994), 525562.

[120] N. Korevaar, R. Schoen. Sobolev spaces and harmonic maps for metric space targets. Comm. Anal. Geom. 1 (1993), 561-659.

[121] V. Kostov. (i) On the Deligne-Simpson problem. C.R.A.S. 329 (1999), 657-662.

(ii) On the Deligne-Simpson problem. Monodromiya v Zadachakh Algebr. Geom. i Differ. Uravn., Tr. Mat. Inst. Steklova 238 (2002), 158-195. 
(iii) On some aspects of the Deligne-Simpson problem. J. Dynam. Control Systems 9 (2003), 393-436.

[122] R. Kulkarni, J. Wood. Topology of nonsingular complex hypersurfaces. Adv. in Math. 35 (1980), 239-263. See also the numerous references given in the Math Reviews MR0563926 of this paper.

[123] J. M. Landsberg. Differential-geometric characterizations of complete intersections. J. Differential Geom. 44 (1996), 32-73.

[124] B. Lasell, M. Ramachandran. Observations on harmonic maps and singular varieties. Ann. Sci. E.N.S. 29 (1996), 135-148.

[125] G. Laumon. (i) La transformation de Fourier géométrique et ses applications. Proceedings of ICM, Kyoto 1990, Math. Soc. Japan (1991), 437-445.

(ii) Sur la cohomologie à supports compacts des variétés de Shimura pour GSp(4) . Compositio Math. 105 (1997), 267-359.

(iii) Manuscript on the Fourier transform between $\mathcal{D}$-modules on an abelian variety and bundles on its universal extension.

[126] G. Laumon, M. Rapoport. The Langlands lemma and the Betti numbers of stacks of $G$-bundles on a curve. Internat. J. Math. 7 (1996), $29-45$.

[127] R. Lazarsfeld. Lengths of periods and Seshadri constants of abelian varieties. Math. Res. Lett. 3 (1996), 439-447.

[128] J. Li, M.S. Narasimhan. Hermitian-Einstein metrics on parabolic stable bundles. Acta Math. Sin. 15 (1999), 93-114.

[129] J. Li, Y.-D. Wang. Existence of Hermitian-Einstein metrics on stable Higgs bundles over open Kähler manifolds. Internat. J. Math. 10 (1999), 1037-1052.

[130] A. Libgober. (i) Alexander polynomial of plane algebraic curves and cyclic multiple planes. Duke Math. J. 49 (1982), 833-851.

(ii) First order deformations for rank one local systems with a nonvanishing cohomology. Arrangements in Boston: a Conference on Hyperplane Arrangements (1999). TOPOLOGY APPL. 118 (2002), 159-168. 
[131] A. Libgober, J. Wood. (i) Diffeomorphic complete intersections with different multidegrees. Bull. Amer. Math. Soc. 2 (1980), 459-461.

(ii) Differentiable structures on complete intersections. II. Singularities, Part 2 (Arcata, 1981), AMS Proc. Sympos. Pure Math. 40 (1983), 123-133.

[132] R. Livné. Appendix to [139].

[133] M. Lönne. On the diffeomorphism groups of elliptic surfaces. Math. Ann. 310 (1998), 103-117.

[134] J. Lurie. On Infinity Topoi. Preprint math.CT/0306109.

[135] E. Markman. (i) Brill-Noether duality for moduli spaces of sheaves on K3 surfaces. J. Alg. Geom. 10 (2001), 623-694.

(ii) On the monodromy of moduli spaces of sheaves on K3 surfaces, I and II. Preprints math.AG/0305042, math.AG/0305043.

(iii) Algebraic geometry, integrable systems, and Seiberg-Witten theory. Integrability: the Seiberg-Witten and Whitham equations eds. H. Braden,

I. Krichever. Gordon and Breach, Amsterdam (1999), 23-41.

[136] E. Markman, E. Xia. The moduli of flat $\mathrm{PU}(p, p)$-structures with large Toledo invariants. Math. Z. 240 (2002), 95-109.

[137] M. Mehta. Birational equivalence of Higgs moduli. Preprint math. AG/0311122.

[138] T. Mochizuki. (i) Asymptotic behaviour of tame nilpotent harmonic bundles with trivial parabolic structure. J. Diff. Geom. 62 (2002), 351559 .

(ii) The Gromov-Witten class and a perturbation theory in algebraic geometry. Amer. J. Math. 123 (2001), 343-381.

(iii) Asymptotic behaviour of tame harmonic bundles and an application to pure twistor $D$-modules. Preprint math.DG/0312230.

[139] B. Moishezon. Complex surfaces and connected sums of complex projective planes. (appendix by R. Livne). Springer L.N.M. 603 (1977).

[140] B. Moishezon, M. Teicher. (i) Braid group technique in complex geometry. I. Line arrangements in $\mathbf{C P}^{2}$. Braids (Santa Cruz, 1986), CoNTEMP. MATH. 78, A.M.S. (1988), 425-555. 
(ii) Braid group technique in complex geometry. II. From arrangements of lines and conics to cuspidal curves. Algebraic geometry (Chicago, 1989), Springer L.N.M. 1479 (1991), 131-180.

(iii) Braid group technique in complex geometry. V. The fundamental group of a complement of a branch curve of a Veronese generic projection. Comm. Anal. Geom. 4 (1996), 1-120.

[141] J. Morgan. The algebraic topology of smooth algebraic varieties. Publ. Math. I.H.E.S. 48 (1978), 137-204.

[142] J. Morgan, T. Mrowka. On the diffeomorphism classification of regular elliptic surfaces. Internat. Math. Res. Notices 6 (1993), 183-184.

[143] I. Mundet i Riera. Yang-Mills-Higgs theory for symplectic fibrations. Preprint math.SG/9912150.

[144] A. Nadel. (i) Singularities and Kodaira dimension of the moduli space of flat Hermitian-Yang-Mills connections. Compositio Math. 67 (1988), 121-128.

(ii) Invariants for holomorphic vector bundles. Math. Ann. 309 (1997), $37-52$.

[145] H. Nakajima. Hyper-Kähler structures on moduli spaces of parabolic Higgs bundles on Riemann surfaces. Moduli of vector bundles (Sanda, Kyoto, 1994) Lect. Notes Pure Appl. Math. 179 (1996), 199-208.

[146] Y. Namikawa, J. Steenbrink. Global smoothing of Calabi-Yau threefolds. Inventiones 122 (1995), 403-419.

[147] T. Napier, M. Ramachandran. The $L^{2} \bar{\partial}$-method, weak Lefschetz theorems, and the topology of Kähler manifolds. J. Amer. Math. Soc. 11 (1998), 375-396.

[148] V. Navarro Aznar.

[149] W. Neumann, P. Norbury. Vanishing cycles and monodromy of complex polynomials. Duke Math. J. 101 (2000), 487-497.

[150] L. Ni, H. Ren. Hermitian-Einstein metrics for vector bundles on complete Kähler manifolds. Trans. Amer. Math. Soc. 353 (2001), 441-456. 
[151] N. Nitsure. (i) Moduli space of semistable pairs on a curve. Proc. London Math. Soc. 62 (1991), 275-300.

(ii) Moduli of semistable logarithmic connections. J. Amer. Math. Soc. 6 (1993), 597-609.

[152] M. Nori. Zariski's conjecture and related problems. Ann. Sci. E.N.S. 16 (1983), 305-344.

[153] K. O'Grady. The weight-two Hodge structure of moduli spaces of sheaves on a K3 surface. J. Alg. Geom. 6 (1997), 599-644.

[154] C. Okonek, A. Teleman. (i) Master spaces and the coupling principle: from geometric invariant theory to gauge theory. Comm. Math. Phys. 205 (1999), 437-458.

(ii) Gauge theoretical equivariant Gromov-Witten invariants and the full Seiberg-Witten invariants of ruled surfaces. Comm. Math. Phys. 227 (2002), 551-585.

[155] A. Otwinowska. Composantes de petite codimension du lieu de Noether-Lefschetz: un argument asymptotique en faveur de la conjecture de Hodge pour les hypersurfaces. J. Alg. Geom. 12 (2003), 307-320.

[156] V. Pati. $L^{2}$-cohomology of algebraic varieties in the Fubini metric. Hodge theory, Sant Cugat, 1985, Springer L.N.M. 1246 (1987), 154164.

[157] T. Pantev. Comparison of generalized theta functions. Duke Math. J. 76 (1994), 509-539.

[158] S. Papadima, A. Suciu. Higher homotopy groups of complements of complex hyperplane arrangements. Adv. Math. 165 (2002), 71-100.

[159] R. Pelissier.Catégories enrichies faibles. Thesis, Université de NiceSophia Antipolis (2002), preprint math.AT/0308246.

[160] O. Penacchio. Structures de Hodge mixtes et fibrés sur le plan projectif complexe. Thesis, Université Paul Sabatier, Toulouse (2002), preprint math. AG/0307156.

[161] G. Perelman. The entropy formula for the Ricci flow and its geometric applications. Preprint math.DG/0211159. 
[162] U. Persson. (i) On degenerations of algebraic surfaces. Mem. Amer. Math. Soc. 11 (1977).

(ii) An introduction to the geography of surfaces of general type. Algebraic geometry, Bowdoin, 1985 A.M.S. Proc. Sympos. Pure Math. 46 (1987), 195-218.

[163] U. Persson, C. Peters. Some aspects of the topology of algebraic surfaces. Proceedings of the Hirzebruch 65 Conference on Algebraic Geometry (Ramat Gan, 1993) Israel Math. Conf. Proc. 9, Bar-Ilan Univ. (1996), 377-392.

[164] U. Persson, C. Peters, G. Xiao. Geography of spin surfaces. Topology 35 (1996), 845-862.

[165] U. Persson, H. Pinkham. Some examples of nonsmoothable varieties with normal crossings. Duke Math. J. 50 (1983), 477-486.

[166] C. Peters. (i) Introduction to the theory of compact complex surfaces. Differential geometry, global analysis, and topology (Halifax, 1990), CMS Conf. Proc. 12, AMS, Providence (1991), 129-156.

(ii) Rigidity for variations of Hodge structure and Arakelov-type finiteness theorems. Compositio Math. 75 (1990), 113-126.

(iii) On Arakelov's finiteness theorem for higher-dimensional varieties. Conference on algebraic varieties of small dimension (Turin, 1985). Rend. Sem. Mat. Univ. Politec. Torino (1987), 43-50.

(iv) Math Reviews MR0466149 (57 \# 6030), review of [162] (i).

[167] D. Quillen. (i) Rational homotopy theory. Ann. of Math. 90 (1969), 205-295.

(ii) Homotopical algebra. Springer L.N.M. 43 (1967).

(iii) Characteristic classes of representations. Algebraic K-theory (Northwestern Univ., Evanston, 1976), Springer L.N.M. 551 (1976), 189-216.

[168] F. Rahmati. Déformation des surfaces projectives toriques. Bull. Soc. Sci. Lett. Lódź Sér. Rech. Déform. 23 (1997), 23-31.

[169] Z. Ran. (i) Deformations of manifolds with torsion or negative canonical bundle. J. Algebraic Geom. 1 (1992), 279-291.

(ii) On the variety of rational space curves. Israel J. Math. 122 (2001), 359-369. 
[170] A. Reznikov. (i) Continuous cohomology of the group of volumepreserving and symplectic diffeomorphisms, measurable transfer and higher asymptotic cycles. Selecta Math. 5 (1999), 181-198.

(ii) Symplectic twistor spaces. Ann. Global Anal. Geom. 11 (1993), 109118.

(iii) Simpson's theory and superrigidity of complex hyperbolic lattices. C. R. A. S. Math. 320 (1995), 1061-1064.

(iv) Characteristic classes in symplectic topology. Selecta Math. 3 (1997), 601-642.

(v) Rationality of secondary classes. J. Diff. Geom. 43 (1996), 674-692.

(vi) All regulators of flat bundles are torsion. Ann. of Math. 141 (1995), 173-186.

(vii) The structure of Kähler groups. I. Second cohomology. Motives, polylogarithms and Hodge theory, Irvine, 1998, Int. Press LeCt. SER. 3 (II) (2002), 717-730.

[171] A. Robb, M. Teicher. Applications of braid group techniques to the decomposition of moduli spaces, new examples. Special issue on braid groups and related topics (Jerusalem, 1995). Topology Appl. 78 (1997), 143-151.

[172] C. Sabbah. (i) Frobenius manifolds: isomonodromic deformations and infinitesimal period mappings. Exposition. Math. 16 (1998), 1-57.

(ii) Harmonic metrics and connections with irregular singularities. Ann. Inst. Fourier 49 (1999), 1265-1291.

(iii) Polarizable twistor $\mathcal{D}$-modules. Preprint, Ecole Polytechnique.

[173] M. Saito. (i) Mixed Hodge modules and admissible variations. C.R.A.S. 309 (1989), 351-356.

(ii) Mixed Hodge modules, Publ. RIMS 26 (1990), 221-335.

[174] W. Schmid. Variation of Hodge structure: the singularities of the period mapping. Inventiones 22 (1973), 211-319.

[175] A. Schmitt. Projective moduli for Hitchin pairs. Internat. J. Math. 9 (1998), 107-118.

[176] H. Schoutens. Bounds in cohomology. Israel J. Math. 116 (2000), 125169. 
[177] C. Simpson. (i) Transcendental aspects of the Riemann-Hilbert correspondence. Illinois J. Math. 34 (1990), 368-391.

(ii) Products of matrices. Differential geometry, global analysis, and topology (Halifax, 1990), CMS Conf. Proc. 12 (1991), 157-185.

(iii) Some families of local systems over smooth projective varieties. Ann. of Math. 138 (1993), 337-425.

(iv) The Hodge filtration on nonabelian cohomology. Algebraic geometry, Santa Cruz 1995 AMS Proc. Symp. Pure Math. 62 (2) (1997), 217-281.

(v) Mixed twistor structures. Preprint alg-geom/9705006.

(vi) Algebraic aspects of higher nonabelian Hodge theory. Motives, polylogarithms and Hodge theory, Irvine, 1998, Int. Press LeCt. Ser. 3 (II) (2002), 417-604.

[178] Y.-T. Siu. Strong rigidity for Kähler manifolds and the construction of bounded holomorphic functions. Discrete Groups and Analysis, Progr. MATH. 67, Birkhäuser (1987), 124-151.

[179] I. Smith. (i) Lefschetz pencils and divisors in moduli space. Geom. Topol. 5 (2001), 579-608.

(ii) Lefschetz fibrations and the Hodge bundle. Geom. Topol. 3 (1999), 211-233.

[180] A. Sommese, A. Van de Ven. Homotopy groups of pullbacks of varieties. Nagoya Math. J. 102 (1986), 79-90.

[181] A. Sommese, J. Verschelde. Numerical homotopies to compute generic points on positive dimensional algebraic sets. Complexity theory, real machines, and homotopy (Oxford, 1999). J. CompleXity 16 (2000), $572-602$.

[182] J. Steenbrink. Some remarks about the Hodge conjecture. Hodge theory, Sant Cugat, 1985, Springer L.N.M. 1246 (1987), 165-175.

[183] J. Steenbrink, S. Zucker. Variation of mixed Hodge structure. I. Inventiones 80 (1985), 489-542.

[184] C. Teleman. Borel-Weil-Bott theory on the moduli stack of $G$-bundles over a curve. Inventiones 134 (1998), 1-57. 
[185] M. Thaddeus. Variation of moduli of parabolic Higgs bundles. J. Reine Angew. Math. 547 (2002), 1-14.

[186] G. Tian. (i) Smoothness of the universal deformation space of compact Calabi-Yau manifolds and its Petersson-Weil metric. Mathematical aspects of string theory (San Diego, 1986) AdV. Ser. Math. Phys. 1 World Scientific (1987), 629-646.

(ii) Smoothing 3-folds with trivial canonical bundle and ordinary double points. Essays on mirror manifolds, 458-479, Internat. Press, Hong Kong, 1992.

(iii) Degeneration of Khler-Einstein manifolds, I. Differential geometry: geometry in mathematical physics and related topics (Los Angeles, 1990) AMS Proc. Sympos. Pure Math. 54 (1993), 595-609.

(iv) Kähler-Einstein metrics with positive scalar curvature. Inventiones 130 (1997), 1-37.

[187] K. Timmerscheidt. Mixed Hodge theory for unitary local systems. $J$. Reine Angew. Math. 379 (1987), 152-171.

[188] A. Todorov. The Weil-Petersson geometry of the moduli space of $S U(n \geq 3)$ (Calabi-Yau) manifolds, I. Comm. Math. Phys. 126 (1989), 325-346.

[189] B. Toen. (i) Vers une interprétation galoisienne de la théorie de l'homotopie. Cah. Topol. Géom. Diff. Catég. 43 (2002), 257-312.

(ii) On motives for Deligne-Mumford stacks. Int. Math. Res. Notices 17 (2000), 909-928.

(iii) Champs affines. Preprint math. AG/0012219.

(iv) Homotopical and Higher Categorical Structures in Algebraic Geometry. Preprint math.AG/0312262.

[190] B. Toen, G. Vezzosi. (i) "Brave New" Algebraic Geometry and global derived moduli spaces of ring spectra. Preprint math.AT/0309145.

(ii) Homotopical Algebraic Geometry I: Topos theory. Preprint math. AG/0207028.

(iii) From HAG to DAG: derived moduli spaces. Preprint math. AG/0210407.

[191] D. Toledo. (i) Representations of surface groups in complex hyperbolic space. J. Differential Geom. 29 (1989), 125-133. 
(ii) Examples of fundamental groups of compact Kähler manifolds. Bull. London Math. Soc. 22 (1990), 339-343.

(iii) Projective varieties with non-residually finite fundamental group. Publ. Math. I.H.E.S. 77 (1993), 103-119.

(iv) Rigidity theorems in Kähler geometry and fundamental groups of varieties. Several complex variables (Berkeley, 1995-1996) MSRI PUBL. 37, Cambridge Univ. Press (1999), 509-533.

[192] K. Uhlenbeck, S.-T. Yau. On the existence of Hermitian-Yang-Mills connections in stable vector bundles. Frontiers of the mathematical sciences: 1985 (New York, 1985). Comm. Pure Appl. Math. 39 (1986), no. S, suppl., S257-S293.

[193] M. Verbitsky.

(i) Tri-analytic subvarieties of hyper-Kaehler manifolds. Geom. Funct. Anal. 5 (1995), 92-104.

(ii) Deformations of trianalytic subvarieties of hyper-Kähler manifolds. Selecta Math. 4 (1998), 447-490.

[194] C. Voisin. (i) Variations of Hodge structure and algebraic cycles. Proc. ICM (Zürich, 1994), Birkhäuser (1995), 706-715.

(ii) Remarks on zero-cycles of self-products of varieties. Moduli of vector bundles (Sanda, Kyoto, 1994), Lecture Notes in Pure And Appl. MAтн. 179 (1996), 265-285.

(iii) Thórie de Hodge et géométrie algébrique complexe. S.M.F. Cours SpéCIALisÉs 10 (2002). English translation by L. Schneps: CAMBridge Studies in Adv. Math. 76, 77, Cambridge University Press (2003).

(iv) A counterexample to the Hodge conjecture extended to Kähler varieties. Int. Math. Res. Not. 20 (2002), 1057-1075.

(v) On the homotopy types of compact kaehler and complex projective manifolds. Preprint math. AG/0312032.

[195] F. Warner. Introduction to manifolds, Scott-Foresman, New York (1971).

[196] M. Wolf. Harmonic maps from surfaces to R-trees. Math. Z. 218 (1995), 577-593.

[197] E. Xia. The moduli of flat $U(p, 1)$ structures on Riemann surfaces. Geom. Dedicata 97 (2003), 33-43. 
[198] Y.-H. Yang. On non-Kählerianity of nonuniform lattices in $S O(n, 1)(n \geq 4)$. Manuscripta Math. 103 (2000), 401-407.

[199] K. Yokogawa. (i) Compactification of moduli of parabolic sheaves and moduli of parabolic Higgs sheaves. J. Math. Kyoto Univ. 33 (1993), 451504.

(ii) Infinitesimal deformation of parabolic Higgs sheaves. Internat. J. Math. 6 (1995), 125-148.

[200] J. Zhang. Threefolds with vanishing Hodge cohomology. Preprint math. AG/0312239.

[201] S. Zucker. (i) Variation of mixed Hodge structure. II. Inventiones 80 (1985), 543-565.

(ii) Hodge theory with degenerating coefficients: $L^{2}$ cohomology in the Poincaré metric. Ann. of Math. 109 (1979), 415-476.

[202] K. Zuo. (i) Factorizations of nonrigid Zariski dense representations of $\pi_{1}$ of projective algebraic manifolds. Inventiones 118 (1994), 37-46.

(ii) Representations of fundamental groups of algebraic varieties. Springer L.N.M. 1708 (1999).

(iii) On the negativity of kernels of Kodaira-Spencer maps on Hodge bundles and applications. Kodaira's issue. Asian J. Math. 4 (2000), 279301. 